\title{
Integral Equations and the Determination of Green's Functions in the Theory of Potential.
}

\author{
By Professor H. S. Carslaw.
}

(Received 20th May 1912. Read 9th December 1919).

In the Theory of Potential the term Green's Function, used in a slightly different sense by Maxwell, now denotes a function associated with a closed surface $S$, with the following properties :-

(i) In the interior of $\mathrm{S}$, it satisfies $\nabla^{2} \mathrm{~V}=0$.

(ii) At the boundary of $S$, it vanishes.

(iii) In the interior of $\mathrm{S}$, it is finite and continuous, as also its first and second derivatives, except at the point $\left(x_{1}, y_{1}, z_{1}\right)$.

(iv) At the point $\left(x_{1}, y_{1}, z_{1}\right)$, it becomes infinite as $\frac{1}{4 \pi r}$, when $r$ tends to zero, $r$ being the distance from $(x, y, z)$ to $\left(x_{1}, y_{1}, z_{1}\right)$.

In other branches of Applied Mathematics similar functions have been introduced and called by the same name. These have become of increasing importance as kernels of Homogeneous Integral Equations. In the applications of Integral Equations in Mathematical Physics they occupy a prominent place.*

* Cf. Kneser, Die Integralgleichungen und ihre Anwendungen in der Mathematischen Physik (Braunschweig, 1911).

We shall refer to Kneser's book in the following pages as Kneser, Integralgleichungen.

Hilbert, Grundzïge einer allgemeinen Theorie der linearen Integralgleichnugen, Göttinger Nachrichten, Math..Phys. Klasse, 1904.

Stekloff, Théorie générale des fonctions fondamentales, Ann. de la Fac. des Sc. de Toulouse (2), T. 6, p. 351, 1904. 
$\S 2$. Let $\left(x_{1}, y_{1}, z_{1}\right)$ be the coordinates of the point $\mathbf{P}$ at which the Green's Function is to be infinite as $\frac{1}{4 \pi r}$.

Let $(x, y, z)$ be the coordinates of any point $\mathrm{Q}$ in the region bounded by $\mathrm{S}$.

Then the Green's Function at $\mathrm{Q}$ will depend upon $(x, y, z)$ and $\left(x_{1}, y_{1}, z_{1}\right)$. Following Kneser, ${ }^{*}$ we shall denote it by $\mathrm{K}(0,1)$, and refer to the points $P$ and $Q$ as $(0)$ and (1) respectively.

Thus we have, inside $\mathrm{S}$,

and

$$
\begin{aligned}
\nabla^{2} \mathrm{~K}(0,1) & =0, \\
\mathrm{~K}(0,1) & =\frac{1}{4 \pi r}+\mathrm{M}(0,1),
\end{aligned}
$$

where $\mathbf{M}(0,1)$ is a finite and continuous function of $(0,1)$ in the given region, its first and second differential coefficients with respect to $x, y$ and $z$ being also finite and continuous there.

Further, $\quad \mathrm{K}(0,1)=0$, on the surface $\mathrm{S}$.

A simple application of Green's Theorem shows that $\mathbf{K}(0,1)$ is a symmetrical function of $(x, y, z)$ and $\left(x_{1}, y_{1}, z_{1}\right)$.

In other words, we have

$$
\mathbf{K}(0,1)=\mathbf{K}(1,0) .
$$

§. Now let $\psi$ be any solution of

$$
\nabla^{2} \psi+\lambda \psi=0 \text {, }
$$

which vanishes on the surface $S$, and with its first and second derivatives, is finite and continuous in the region bounded by $\mathrm{S}$.

Applying Green's Theorem to the region between $\mathbf{S}$ and a small sphere $\Sigma$, whose centre is at the point (1), we have

$$
\begin{aligned}
& \iiint\left\{\psi \nabla^{2} \mathrm{~K}(0,1)-\mathrm{K}(0,1)\right.\left.\nabla^{2} \psi\right\} d v \\
&+\iint\left\{\psi \frac{\partial}{\partial} \mathrm{K}(0,1)-\mathrm{K}(0,1) \frac{\partial}{\partial n} \psi\right\} d \Sigma=0,
\end{aligned}
$$

where $\frac{\partial}{\partial n}$ denotes differentiation along the outward normal to $\Sigma$, and $d v$ the element of volume at the point $(0)$.

* Cf. Kneser, Integralgleichungen, p. 127. 
When the radius of $\Sigma$ tends to zero, we obtain the known result

$$
\psi(1)=\lambda \iiint \mathrm{K}(0,1) \psi(0) d v,
$$

where $\psi(0)$ stands for $\psi(x, y, z)$ and $\psi(1)$ for $\psi\left(x_{1}, y_{1}, z_{1}\right)$.

Therefore the functions $\psi$ are characteristic functions (Eigenfunktionen), and the numbers $\lambda$ are characteristic numbers (Eigenwerte) of a Homogeneous Integral Equation with $\mathrm{K}(0,1)$ as its symmetrical kernel.*

$\S 4$. These characteristic functions are supposed to be normalised, so that

$$
\iiint \psi^{2} d v=1
$$

the integration being taken through the interior of $\mathrm{S}$.

If we assume that $K(0,1)$ can be expanded in an infinite series

$$
\mathrm{A}_{1} \psi_{1}+\mathrm{A}_{2} \psi_{2}+\ldots
$$

which can be integrated term by term, on multiplying by $\psi_{n}$ and integrating, we find that

$$
\mathbf{A}_{n} \iiint \psi_{n}^{2} d v=\iiint \mathbf{K}(0,1) \psi_{n}(0) d v .
$$

Therefore with this hypothesis we would have

$$
\mathbf{A}_{n}=\iiint \mathbf{K}(0,1) \psi_{n}(0) d v,
$$

and

$$
\mathbf{K}(0,1)=\frac{\psi_{1}(0) \psi_{2}(1)}{\lambda_{1}}+\frac{\psi_{2}(0) \psi_{2}(1)}{\lambda_{2}}+
$$

We are thus led to enquire whether the series in (2) really converges and represents the function $K(0,1)$.

$\S 5$. For the case of a finite and continuous symmetrical kernel, $\mathrm{K}(x, y)$, it was shown by Hilbert and Schmidt $f$ that this equality (2) hoids, provided the series is uniformly convergent with regard to both variables in the given interval.

* Cf. Bocher, An Introduction to the Study of Integral Equations (Camb. Tracts, No. 10), p. 57.

$\dagger$ Cf. Schmidt, Zur Theorie der linearen und nichtlinearen Integralgleichungen, Math. Ann. Bd. 63, p. 449, 1907. 
Kneser has extended* the theorem to kernels which become infinite as $\frac{1}{r}$, and proved that the Green's Function, as defined in $\S 1$, is given by the infinite series

$$
\frac{\psi_{1}(0) \psi_{1}(1)}{\lambda_{1}}+\frac{\psi_{2}(0) \psi_{2}(1)}{\lambda_{2}}+\ldots
$$

provided that the series converges uniformly within $\mathrm{S}$, both with respect to $(x, y, z)$ and $\left(x_{1}, y_{1}, z_{1}\right)$, when the distance $r_{01}$ between $(0)$ and (1) remains greater than some fixed quantity.

In some cases it is not difficult to prove the uniform convergence of the series, so that the Green's Functions are definitely established by means of this theorem. Various examples of this are given in Kneser's book.

In other cases, however, we have to be content with the position that the Integral Equation (1) suggests the expression for the Green's Function, which has then to be verified independently.

It should be noted that in these cases, repeated series, in which the summations are taken one after the other, are substituted for the original double, or triple, series. $\dagger$

In the following pages we shall show that the series (2) does give the Green's Function in the Theory of Potential, when the region is bounded by concentric circular cylinders, planes perpendicular to the axis, and axial planes: and by concentric spheres, axial planes and cones. The Green's Functions for these cases have been obtained by other methods by Dougall. I They can also be deduced from results given by me§ for Instantaneous Point Sources in the Conduction of Heat, by replacing the instantaneous source by a constant and continuous one.

* Kneser, Die Theore der Integralgleichungen und die Darstellung willkürlicher Funktionen in der mathematischen Physik, Math. Ann. Bd. 63, p. 486, 1907. Also Integralgleichungen, $\$ 41$.

+ Cf. Kneser, Integralgleichungen und Darstellung willkürlicher Funktionen von zwei Variabeln, Rend. Circ. Mat. di Palermo, T. XXVII., p. 117, 1909.

‡ Dougall, The Determination of Green's Functions by means of Cylindrical and Spherical Harmonics, Proc. Edin. Math. Soc., Vol. XVIII., p. 33, 1900.

\$ The Green's Functions for a Wedge of any Angle and other Problems in the Conduction of Heat, Proc. Lond. Math. Soc. (2), Vol. VIII., p. 365, 1910. 
$\S 6$. The following theorems dealing with Bessel's Functions will be required later:-

I. Let $\rho_{1}, \rho_{3}, \ldots$ be the positive roots of the equation

$$
\mathrm{J}_{n}(\rho a)=0 . \quad(n>0) .
$$

Then $\quad \sum_{\rho} \frac{\mathbf{J}_{n}(\rho x) J_{n}(\rho \xi)}{\rho^{2} \int_{0}^{a} x J_{n}^{2}(\rho x) d x}=\frac{1}{2 n}\left(\frac{x}{a}\right)^{n}\left\{\left(\frac{a}{\xi}\right)^{n}-\left(\frac{\xi}{a}\right)^{n}\right\}$ for $0<x<\xi$

$$
=\frac{1}{2 n}\left(\frac{\xi}{a}\right)^{n}\left\{\left(\frac{a}{x}\right)^{n}-\left(\frac{x}{a}\right)^{n}\right\} \text { for } \xi<x<a .^{*}
$$

II. Let $\rho_{1}, \rho_{2}, \ldots$ be the positive roots of the equation

$$
\begin{gathered}
\mathrm{J}_{n}(\rho a) \mathbf{K}_{n}(i \rho b)-\mathrm{J}_{u}(\rho b) \mathbf{K}_{n}(i \rho a)=0,0<a<b, \dagger \\
n>0,
\end{gathered}
$$

and let $\mathrm{U}_{n}(\rho x)$ stand for the expression

$$
\mathrm{J}_{n}(\rho x) \mathbf{K}_{n}(i \rho b)-\mathbf{J}_{n}(\rho b) \mathbf{K}_{n}(i \rho x) .
$$

Then

$\sum_{\rho} \frac{\mathrm{L}_{n}(\rho x) \mathrm{U}_{n}(\rho \xi)}{\rho^{2} \int_{a}^{b} x \mathrm{U}_{n}^{2}(\rho x) d x}=\frac{\frac{1}{2 n}\left\{\left(\frac{x}{a}\right)^{n}-\left(\frac{a}{x}\right)^{n}\right\}\left\{\left(\frac{b}{\xi}\right)^{n}-\left(\frac{\xi}{b}\right)^{n}\right\}}{\left(\frac{b}{a}\right)^{n}-\left(\frac{a}{b}\right)^{n}}$

for $a<x<\xi<b$,

$$
=\frac{\frac{1}{2 n}\left\{\left(\frac{\xi}{a}\right)^{n}-\left(\frac{a}{\xi}\right)^{n}\right\}\left\{\left(\frac{b}{x}\right)^{n}-\left(\frac{x}{b}\right)^{n}\right\}}{\left(\frac{b}{a}\right)^{n}-\left(\frac{a}{b}\right)^{n}}
$$

for $a<\xi<x<b$.

The second of these theorems can be proved by an argument similar to that by which Kneser establishes the first. The value

* Cf. Kneser, Integralgleichungen, $\$ 27$.

† As usual $\mathrm{K}_{n}(i x)$ stands for $\frac{\pi}{2 \sin n \pi} e^{-\frac{1}{n n i \pi}}\left(\underset{-n}{\mathrm{~J}}(x)-e^{n i \pi} \mathrm{J}_{n}(x)\right)$. 
of $U_{n}(x)$ for large values of $x$ is needed, and the fact that, when $\rho$ is large, the root $\rho_{d}$ of the equation

$$
J_{n}(\rho a) \mathrm{K}_{n}(i \rho b)-J_{n}(\rho b) \mathrm{K}_{n}(i \rho a)=0
$$

is approximately $\frac{s a \pi}{b-a}$. $*$

III. If $\rho_{2} \rho^{\prime}$ are two different positive roots of

$$
\begin{array}{r}
\mathrm{U}_{n}(\rho a)=\mathrm{J}_{n}(\rho a) \mathrm{K}_{n}(i \rho b)-\mathrm{J}_{n}(\rho b) \mathrm{K}_{n}(i \rho a)=0, n>0 \\
\text { (i) } \int_{a}^{b} x \mathrm{U}_{n}(\rho x) \mathrm{U}_{n}\left(\rho^{\prime} x\right) d x=0 \\
\text { and (ii) } \int_{a}^{b} x \mathrm{U}_{n}^{2}(\rho x) d x=-\frac{a}{2 \rho} \frac{d}{d \rho} \mathrm{U}_{n}(\rho a) \frac{d}{d a} \mathrm{U}_{n}(\rho a)
\end{array}
$$

The proof of this theorem offers no difficulty. +

\section{Cylindrical Coordinates.}

$\$ 7$. We proceed to examine the different series

$$
\frac{\psi_{1}(0) \psi_{1}(1)}{\lambda_{1}}+\frac{\psi_{2}(0) \psi_{2}(1)}{\lambda_{2}}+\ldots
$$

for the regions named in $\$ 5$.

We begin with those in which cylindrical coordinates $(r, \theta, z)$ are used. With these coordinates the equation

becomes

$$
\nabla^{2} \psi+\lambda \psi=0
$$

$$
\frac{\partial^{2} \psi}{\partial r^{2}}+\frac{1}{r} \frac{\partial \psi}{\partial r}+\frac{1}{r^{2}} \frac{\partial^{2} \psi}{\partial \theta^{2}}+\frac{\partial^{2} \psi}{\partial z^{2}}+\lambda \psi=0
$$

Space bounded by the cylinder $r=a$, and the planes $z=0, z=c$.

The finite and continuous solutions vanishing at the boundary are given by

$$
\psi_{\rho m n}=\mathrm{A}_{\rho m n} \mathrm{~J}_{m}(\rho r)_{\sin }^{\cos } m \theta \sin \frac{n \pi}{c} z
$$

where $m, n$ are positive integers, including zero, $\rho$ is a positive root of $\mathrm{J}_{m}(\rho a)=0$, and $\lambda=\rho^{2}+\frac{n^{2} \pi^{2}}{c^{2}}$.

* Cf. Gray and Mathews, Bessel Functions, p. 242.

+ Cf. Carslaw, Fourier's Series, p. 315. 
To normalise these functions we choose $A_{\rho m n}$ so that

$$
\mathrm{A}_{\rho m n}^{2} \int_{0}^{a} r \mathrm{~J}_{m}^{2}(\rho r) d r \int_{0}^{2 \pi} \cos ^{2} \sin ^{2} m \theta d \theta \int_{0}^{c} \sin ^{2} \frac{n \pi}{c} z d z=1 .
$$

Thus

$$
\mathbf{A}_{\rho m n}^{2}=\frac{a_{m}}{\pi c \int_{0}^{a} r \mathbf{J}_{m}^{2}(\rho r) d r},
$$

where $a_{0}=1$ and $a_{m}=2$, for $m \geqq 1$.

Therefore the series corresponding to

is

$$
\Sigma \frac{\psi_{s}(0) \psi_{s}(1)}{\lambda_{s}}
$$

$$
\frac{1}{\pi c} \sum_{\rho} \sum_{m} \sum_{n} \frac{a_{m} \mathrm{~J}_{m}(\rho r) \mathrm{J}_{m}\left(\rho r^{\prime}\right)}{\left(\rho^{2}+\frac{n^{2} \pi^{2}}{c^{2}}\right) \int_{0}^{a} r \mathrm{~J}_{m}^{2}(\rho r) d r} \cos m\left(\theta-\theta^{\prime}\right) \sin \frac{n \pi}{c} z \sin \frac{n \pi}{r} z^{\prime} .
$$

Now it is easy to show that

$$
\left.\begin{array}{rl}
\frac{2}{c} \sum_{1}^{\infty} \frac{\sin \frac{n \pi}{c} z \sin \frac{n \pi}{c} z^{\prime}}{\rho^{2}+\frac{n^{2} \pi^{2}}{c^{2}}} & =\frac{1}{\rho} \frac{\sinh \rho(c-z) \sinh \rho z^{\prime}}{\sinh \rho c}, \text { for } 0<z^{\prime}<z: \\
& =\frac{1}{\rho} \frac{\sinh \rho\left(c-z^{\prime}\right) \sinh \rho z}{\sinh \rho c}, \text { for } z<z^{\prime}<c
\end{array}\right\} .
$$

Therefore if we start with a repeated series, and sum first with regard to $n$, we have

$\sum_{m} a_{m} \cos m\left(\theta-\theta^{\prime}\right) \sum_{\rho} \frac{J_{m}(\rho r) J_{m}\left(\rho r^{\prime}\right)}{2 \pi \rho \int_{0}^{a} r J_{m}^{2}(\rho r) d r} \frac{\sinh \rho(c-z) \sinh \rho z^{\prime}}{\sinh \rho c}$

for $z>z^{\prime}$, and when $z<z^{\prime}$, we have to interchange $z$ and $z^{\prime}$ in (5).

But it is known* that

$$
\int_{0}^{n} r J_{m}^{2}(\rho r) d r=\frac{a^{2}}{2}\left[J_{m}^{\prime}(\rho a)\right]^{2}
$$

when $\rho$ is a root of $J_{m}(\rho a)=0$.

Therefore we can replace (5) by

$\frac{1}{\pi a^{2}} \check{m}_{m}^{\prime} a_{m} \cos m\left(\theta-\theta^{\prime}\right) \sum_{\rho} \frac{J_{m}(\rho r) J_{m}\left(\rho r^{\prime}\right)}{\rho\left[\bar{J}_{m}^{\prime}(\rho a)\right]^{2}} \frac{\sinh \rho(c-z) \sinh \rho z^{\prime}}{\sinh \rho c}$ for $z>z^{\prime} .(6)$

This result agrees with Dougall, loc. cit. $\$ 22(2)$. +

* Cf. Fourier's Series, p. 315.

in In comparing Dougall's results with those given in this paper, it has to be remembered that his Green's Functions are infinite as $\frac{1}{r}$, not as $\frac{1}{4 \pi r}$. 
Corollary. We can deduce from (6) the Green's Functions for

(i) The space bounded by a cylinder $r=a$ :

(ii) The space between the planes $z=0$ and $z=c$.

In (6) change the origin to the point on the axis half-way between the planes.

Then we have

$$
\begin{aligned}
\mathbf{K}(0,1)=\frac{1}{\pi a^{2}} \sum_{m} & a_{m} \cos m\left(\theta-\theta^{\prime}\right) \sum_{\rho} \frac{J_{m}(\rho r) J_{m}\left(\rho r^{\prime}\right)}{\rho\left[J_{m}^{\prime}(\rho u)\right]^{2}} \\
& \frac{\sinh \rho\left(\frac{c}{2}-z\right) \sinh \rho\left(\frac{c}{2}+z^{\prime}\right)}{\sinh \rho c} \text { for }-\frac{c}{2}<z^{\prime}<z<\frac{c}{2} .
\end{aligned}
$$

Put $c=\infty$, and we obtain the Green's Function for the cylinder, $\mathrm{K}(0,1)=\frac{1}{2 \pi a^{2}} \sum_{m} a_{m} \cos m\left(\theta-\theta^{\prime}\right) \sum_{\rho} \frac{\mathrm{J}_{m}(\rho r) J_{m}\left(\rho r^{\prime}\right)}{\rho\left[\mathrm{J}_{m}^{\prime}(\rho a)\right]^{2}} e^{-\rho\left(z-z^{\prime}\right)}$ for $z>z^{\prime} \ldots$ (6).

For the space between the two parallel planes, we put $a=\infty$ in

Using the asymptotic value* of $J_{m}(\rho a)$, namely,

$$
\frac{2}{\sqrt{\pi \rho a}} \cos \left(\frac{m \pi}{2}+\frac{\pi}{4}-\rho a\right)
$$

we have

$$
\mathrm{J}^{\prime}{ }_{i n}(\rho a)=\sqrt{\frac{2}{\pi \rho a}} \sin \left(\frac{m \pi}{2}+\frac{\pi}{4}-\rho a\right) .
$$

If $\rho, \rho+\delta \rho$ are consecutive roots of

we have

$$
\mathbf{J}_{m}(\rho a)=0,
$$

$$
\begin{aligned}
& a \delta \rho=\pi \\
& a^{2}\left[\mathrm{~J}^{\prime}{ }_{m}(\rho a)\right]^{2}=\frac{2 a}{\pi \rho}=\frac{2}{\rho \delta \rho} .
\end{aligned}
$$

Thus from (6)

$$
\begin{aligned}
\mathbf{K}(0,1) & =\frac{1}{2 \pi} \underset{m}{\sum} a_{m} \cos m\left(\theta-\theta^{\prime}\right) \int_{0}^{\infty} J_{m}(\rho r) J_{m}\left(\rho r^{\prime}\right) \frac{\sinh \rho(c-z) \sinh \rho z^{\prime}}{\sinh \rho c} d \rho, \\
& =\frac{1}{2 \pi} \int_{0}^{\infty} \frac{\sinh \rho(c-z) \sinh \rho z^{\prime}}{\sinh \rho c} \mathrm{~J}_{0}(\rho \mathbf{R}) d \rho, \text { for } z>z^{\prime} . \ldots \ldots \ldots \ldots . . . .
\end{aligned}
$$

* Cf. Macdonald, The Electrical Distribution on a Conductor bounded by Two Spherical Surfaces cutting at any Angle, Proc. Lond. Math. Soc., (1) Vol. XXVI., p. 159, 1895. 
\$8. Space bounded by a cylinder $r=a$; two planes $z=0, z=c$; and two axial planes $\theta=0, \theta=\alpha$.

Here we have $\psi_{\rho m n}=\frac{2}{\sqrt{\alpha c}} \frac{\mathrm{J}}{\alpha}(\beta r) \frac{\sin \frac{m \pi}{\alpha} \theta \sin \frac{n \pi}{c} z}{\left[\int_{0}^{a} r \mathrm{~J}^{2}{ }_{\frac{m \pi}{a}}(\rho r) d r\right]^{\frac{1}{2}}}$,

the function having been normalised.

In this expression, $m$ and $n$ are positive integers ; $\rho$ is a positive root of $\mathrm{J}_{\frac{m \pi}{a}}(\rho a)=0$; and $\lambda=\rho^{2}+\frac{n^{2} \pi^{2}}{c^{2}}$.

Therefore the series corresponding to

$$
\Sigma \frac{\psi_{s}(0)}{\lambda_{s}}
$$

is

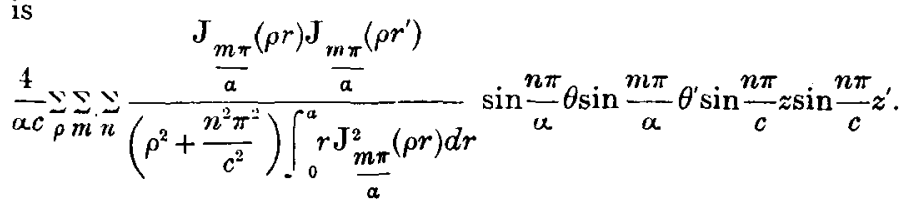

If we sum first with regard to $z$, as in $\$ 7$, we have

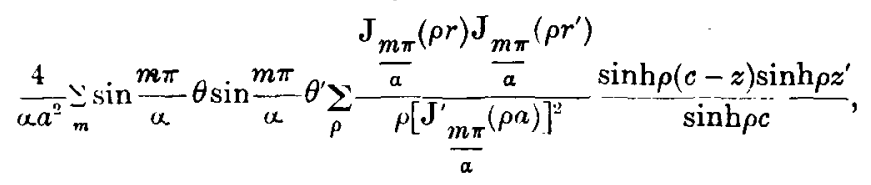

$$
\text { for } z>z^{\prime} \text {. }
$$

This agrees with Dougall, loc. cit. $\$ 25(1)$.

Corollary. We can deduce from (9) the Green's Functions for

(i) The space bounded by a cylinder and two axial planes:

(ii) The wedge of angle $\alpha$ :

(iii) The wedge of angle $\alpha$ and two planes $z=0, z=c$

For these we have, respectively,

$$
\mathrm{K}(0,1)=\frac{2}{\alpha a^{2}} \sum_{m} \sin \frac{m \pi}{\alpha} \theta \sin \frac{m \pi}{\alpha} \theta^{\prime} \sum_{\rho} \frac{\frac{J_{m \pi}}{\rho}(\rho r) \mathrm{J}_{\frac{m \pi}{a}}\left(\rho r^{\prime}\right)}{\frac{\frac{\int_{0}^{u}}{a} \mathrm{~J}_{0}^{2}}{\frac{m \pi}{a}}(\rho r) d r} e^{-\rho\left(z-z^{\prime}\right)}
$$

for $z>z^{\prime}:(10)$ 


$$
\begin{array}{r}
\mathbf{K}(0,1)=\frac{1}{\alpha} \sum_{m} \sin \frac{m \pi}{\alpha} \theta \sin \frac{m \pi}{\alpha} \theta^{\prime} \int_{0}^{\infty} \mathbf{J}_{\frac{m \pi}{\alpha}}(\rho r) \mathbf{J} \frac{m \pi}{a}\left(\rho r^{\prime}\right) e^{-\rho\left(z-z^{\prime}\right)} d \rho \\
\text { for } z>z^{\prime}:
\end{array}
$$

$$
\begin{aligned}
K(0,1)=\frac{2}{\alpha} \Sigma_{m} \sin \frac{m \pi}{\alpha} \theta \sin \frac{m \pi}{\alpha} \theta^{\prime} & \int_{0}^{\infty} J_{\frac{m \pi}{\alpha}}(\rho r) J_{\frac{m \pi}{\alpha}}\left(\rho r^{\prime}\right) \\
& \frac{\sinh \rho(c-z) \sinh \rho z^{\prime}}{\sinh \rho c} d \rho \text { for } z>z^{\prime} .
\end{aligned}
$$

\$9. Space bounded by two cylinders $r=a, r=b$ : and two planes $z=0, z=c$.

Here the normalised function

$$
\psi_{\rho m n}=\sqrt{\frac{a_{m}}{\pi c}} \frac{\mathrm{U}_{m}(\rho r)^{\cos } m \theta \sin { }^{n \pi} z}{\left[\int_{a}^{b} r \mathrm{U}_{m}^{2}(\rho r) d r\right]^{\frac{1}{2}}},
$$

where $\boldsymbol{m}, \boldsymbol{n}$ are positive integers, or zero :

$$
\mathrm{U}_{m}(\rho r)=\mathrm{J}_{m}(\rho r) \mathbf{K}_{m}(i \rho b)-\mathrm{J}_{m}(\rho b) \mathbf{K}_{m}(i \rho r) \text { : }
$$

$\rho$ is a positive root of $U_{m}(\rho a)=0$ :

$$
a_{0}=1, a_{m}=2 \text { for } m \geqq 1 \text { : }
$$

and

$$
\lambda=\rho^{2}+\frac{n^{2} \pi^{2}}{c^{2}} \text {. }
$$

Thus the series corresponding to

$$
\Sigma \frac{\psi_{s}(0) \psi_{s}(1)}{\lambda_{s}}
$$

is

$$
\frac{1}{\pi c} \sum_{\rho=n} \sum \frac{a_{m} \mathrm{U}_{m}(\rho r) \mathrm{U}_{m}\left(\rho r^{\prime}\right)}{\left(\rho^{2}+\frac{n^{2} \pi^{2}}{c^{2}}\right) \int_{a}^{b} r \mathrm{U}_{m}^{2}(\rho r) d r} \cos m\left(\theta-\theta^{\prime}\right) \sin \frac{n \pi}{c} z \sin \frac{n \pi}{c} z^{\prime} .
$$

If we sum first with regard to $n$, we have

$$
\frac{1}{2 \pi} \sum_{m} a_{m} \cos m\left(\theta-\theta^{\prime}\right) \sum_{\rho} \frac{\mathrm{U}_{m}(\rho r) \mathrm{U}_{m}\left(\rho r^{\prime}\right)}{\rho \int_{a}^{b} r \mathrm{U}_{m}^{2}(\rho r) d r} \frac{\sinh \rho(c-z) \sinh \rho z^{\prime}}{\sinh \rho c} \text { for } z>z^{\prime}
$$


Using the result of $\$ 6$ (III.), we have

$$
\begin{aligned}
& -\frac{1}{\pi} \sum_{m} a_{m} \cos m\left(\theta-\theta^{\prime}\right) \underset{\rho}{\sum} \frac{\mathrm{U}_{m}(\rho r) \mathrm{U}_{m}\left(\rho r^{\prime}\right)}{\frac{d}{d \rho} \mathrm{U}_{m}(\rho a) \frac{d}{d a} \mathrm{U}_{n}(\rho a)} \frac{\sinh \rho(c-z) \sinh \rho z^{\prime}}{\sinh \rho c} \\
& \text { for } z>z^{\prime} \text {. }
\end{aligned}
$$

Corollary. For the space bounded by two cylinders we find from (14)

$$
\mathrm{K}(0,1)=-\frac{1}{2 \pi} \sum a_{m} \cos m\left(\theta-\theta^{\prime}\right) \sum_{\rho} \frac{\mathrm{U}_{m}(\rho r) \mathrm{U}_{m}\left(\rho r^{\prime}\right)}{\frac{d}{d \rho} \mathrm{U}_{m}(\rho a) \frac{d}{d a} \mathrm{U}_{m}(\rho a)} e^{-\rho\left(z-z^{\prime}\right)}
$$

\$10. Space bounded by two cylinders $r=a, r=b$ : two planes $z=0, z=c$ : and two axial planes $\theta=0, \theta=\alpha$.

Here the normalised function

$$
\psi_{\rho m n}=\frac{2}{\sqrt{\alpha c}} \frac{U_{\frac{m \pi}{a}}(\rho r) \sin \frac{m \pi}{\alpha} \theta \sin \frac{n \pi}{c} z}{\left[\int_{a}^{b} r \mathrm{U}_{\frac{m \pi}{a}}^{2}(\rho r) d r\right]^{\frac{1}{2}}},
$$

where $\quad \mathrm{U}_{\frac{m \pi}{a}}(\rho r)=\mathrm{J}_{\frac{m \pi}{a}}(\rho r) \mathrm{K}_{\frac{m \pi}{a}}(i \rho b)-\mathrm{J}_{\frac{m \pi}{a}}(\rho b) \mathbf{K}_{\frac{m \pi}{a}}(i \rho r)$ :

$m, n$ are positive integers :

$\rho$ is a positive root of $\mathrm{U}_{\frac{m \pi}{a}}(\rho a)=0$ :

and

$$
\lambda=\rho^{2}+\frac{n^{2} \pi^{2}}{c^{2}} .
$$

Thus the series corresponding to

$$
\Sigma \frac{\psi_{s}(0)}{\lambda_{s}} \underline{\psi_{s}(1)}
$$

is

$$
\frac{4}{\alpha c} \sum_{\rho m n} \sum \frac{\frac{m \pi}{\alpha}(\rho r) \mathrm{U}_{\frac{m \pi}{\alpha}}\left(\rho r^{\prime}\right)}{\left(\rho^{2}+\frac{n^{2} \pi^{2}}{c^{2}}\right) \int_{a}^{b} r U_{\frac{m \pi}{\alpha}}^{2}(\rho r) d r} \sin \frac{m \pi}{\alpha} \theta \sin \frac{m \pi}{\alpha} \theta^{\prime} \sin \frac{n \pi}{c} \approx \sin \frac{n \pi}{c} z^{\prime} .
$$


If we sum first with regard to $n$, we have.

$$
-\frac{4}{a \alpha} \sum_{m} \sin \frac{m \pi}{\alpha} \theta \sin \frac{m \pi}{\alpha} \theta^{\prime} \sum_{\rho} \frac{\mathrm{U}_{\frac{m \pi}{a}}(\rho r) \mathrm{U}_{\frac{m \pi}{a}}\left(\rho r^{\prime}\right)}{\frac{d}{a} \mathrm{U}_{\frac{m \pi}{a}}(\rho a) \frac{d}{d a} \mathrm{U}_{\frac{m \pi}{a}}(\rho a)} \frac{\sinh \rho(c-z) \sinh \rho z^{\prime}}{\sinh \rho c}
$$

Dougall, loc. cit. $\$ 26(2)$ obtains a different result.

\section{Spherical Coordinates.}

$\$ 11$. Before dealing with the regions bounded by spheres, axial planes and cones, it will be necessary to state some theorems regarding the Spherical Harmonics we employ.

Following Hobson* we define $\mathrm{P}_{n}^{-m}(\mu)$ by the equation

$$
\mathrm{P}_{n}^{-m}(\mu)=\frac{1}{\Pi(m)}\left(\frac{1-\mu}{1+\mu}\right)^{\frac{m}{2}} \mathrm{~F}\left(n+1,-n, m+1, \frac{1-\mu}{2}\right) .
$$

This solution of the equation

$$
\frac{d}{d \mu}\left(\left(1-\mu^{2}\right) \frac{d \mathrm{P}}{d \mu}\right)+\left(n(n+1)-\frac{m^{2}}{1-\mu^{2}}\right) \mathrm{P}=0
$$

is finite at $\mu=1(\theta=0)$, and is suitable for the potential problems to be discussed.

I. When $m$ is any positive number, and $n, n^{\prime}$ are roots of

then (i)

$$
\mathbf{P}_{n}^{-m}\left(\mu_{0}\right)=0 \text {, }
$$

$$
\int_{\mu_{0}}^{1} \mathbf{P}_{n}^{-m}(\mu) \mathbf{P}_{n^{\prime}}^{-m}(\mu) d \mu=0
$$

and (ii)

$$
\int_{\mu_{0}}^{1}\left[\mathrm{P}_{n}^{-m}(\mu)\right]^{2} d \mu=-\frac{\left(1-\mu_{0}^{2}\right)}{2 n+1} \frac{d}{d n} \cdot \mathrm{P}_{n}^{-m}\left(\mu_{0}\right) \frac{d}{d \mu_{0}} \cdot \mathrm{P}_{n}^{-m}\left(\mu_{0}\right)
$$

The proof of this theorem resembles that given in Macdonald's Electric Waves, p. 89, for a special case.

* Hobson, On a Type of Spherical Harmonics of Unrestricted Degree, Order, and Argument, Phil. Trans. (A), Vol. 187, p. 451, 1886. 
II. If $m$ is any positive number, and $\pi-\theta=\epsilon$ where $\epsilon$ is small, the positive roots of

are given by

$$
\mathrm{P}_{n}^{m}(\mu)=0
$$

$$
n=m+s+\frac{\Pi(2 m+8)}{\Pi(m) \Pi(m-1) \Pi(s)} \tan ^{2 m} \frac{\epsilon}{2},
$$

where $s$ has all positive integral values, including zero.

In the limit when $\epsilon \rightarrow 0, n=m+s$.

This theorem is due to Macdonald.*

III. If $n$ is any positive root of $\mathrm{P}_{n}^{-m}(\mu)=0$ and $m$ is any positive number,

$$
\left(1-\mu^{2}\right) \frac{d}{d n} \mathrm{P}_{n}^{-m}(\mu)=\frac{2}{\pi \mathrm{P}_{n}^{-m}(-\mu)} \frac{\Pi(n-m)}{\Pi(n+m)} \sin (n-m) \pi .
$$

This theorem is proved in Dougall's paper, $\S 11$, but his proof needs modification for the case of $m$ zero or a positive integer.

IV. If $n$ is any positive root of $\mathrm{P}_{n}^{-m}(\mu)=0$ and $m$ is any positive number, or zero,

$$
\underset{\mu \rightarrow-1}{\text { Lt. }}\left[\left(1-\mu^{2}\right) \frac{d}{d n}\left(\mathbf{P}_{n}^{-m}(\mu) \frac{d}{d \mu} \mathbf{P}_{n}^{-m}(\mu)\right]=-2 \frac{\Pi(n-m)}{\Pi(n+m)} .\right.
$$

Starting from the equation

$$
\mathrm{P}_{n}^{-m}(\mu)=\frac{\sin n \pi}{\sin m \pi} \mathrm{P}_{n}^{-m}\left(\mu^{\prime}\right)-\frac{\Pi(n-m)}{\Pi(n+m)} \frac{\sin (n-m) \pi}{\sin m \pi} \mathrm{P}_{n}^{m}\left(\mu^{\prime}\right),
$$

where $\mu=-\mu^{\prime}$, [cf. Macdonald, Proc. Lond. Math. Soc., (1) Vol. XXXI., p. 273] this theorem follows from an argument similar to that used by Macdonald, [cf. Electric Waves, $\S 63$ ]. The result in (III.) is required in this proof.

V. If $n, n^{\prime}$ are any two positive roots of the equation

$$
\mathbf{P}_{n}^{-m}\left(\mu_{1}\right) \mathbf{P}_{n}^{m}\left(\mu_{0}\right)-\mathbf{P}_{n}^{m}\left(\mu_{1}\right) \mathbf{P}_{n}^{-m}\left(\mu_{0}\right)=0, m>0
$$

and

$$
\mathbf{S}_{n}^{m}(\mu)=\mathbf{P}_{n}^{-m}(\mu) \mathbf{P}_{n}^{m}\left(\mu_{0}\right)-\mathbf{P}_{n}^{m}(\mu) \mathbf{P}_{n}^{-m}\left(\mu_{0}\right) \text {, }
$$

then

$$
\int_{\mu_{0}}^{\mu_{1}} \mathrm{~S}_{n}^{m}(\mu) \mathrm{S}_{n^{\prime}}^{m}(\mu) d \mu=0
$$

and $\quad(2 n+1) \int_{\mu_{0}}^{\mu_{1}}\left[\mathrm{~S}_{n}^{m}(\mu)\right]^{2} d \mu=\left[\left(1-\mu^{2}\right) \frac{d}{d n} \mathrm{~S}_{n}^{m}(\mu) \frac{d}{d \mu} \mathrm{S}_{n}^{m}(\mu)\right]_{\mu_{0}}^{\mu_{1}}$

* Macdonald, Zeroes of the Spherical Harmonic $\mathrm{P}_{n}^{m}(\mu)$ considered as a function of n, Proc. Lond. Math. Soc. (1), Vol. XXXI., p. 273, 1899. 
\$12. Space bounded by the sphere $r=a$.

In Spherical Coordinates $(r, \theta, \phi)$, the equation

becomes

$$
\nabla^{2} \psi+\lambda \psi=0
$$

$$
\frac{\partial^{2} \psi}{\partial r^{2}}+\frac{2}{r} \frac{\partial \psi}{\partial r}+\frac{1}{r^{2}} \frac{\partial}{\partial \mu}\left(\left(1-\mu^{2}\right) \frac{\partial \psi}{\partial \mu}\right)+\frac{1}{r^{2}} \frac{\partial^{2} \psi}{\partial \phi^{2}}+\lambda \psi=0 .
$$

Thus we have

$$
\psi_{\rho m n}=\mathrm{A}_{\rho m n} \frac{\mathrm{J}_{n+\frac{j}{3}}(\rho r)}{\sqrt{r}} \mathrm{P}_{n}^{m}(\mu)_{\sin ^{m}}^{\cos } \phi,
$$

when $m$ is any positive integer, or zero:

$n$ is any positive integer :

$\rho$ is any positive root of $J_{n+\frac{1}{3}}(\rho a)=0$ :

and $\lambda=\rho^{2}$.

The function having been normalised, we find that the series corresponding to

is

$$
\frac{\psi_{1}(0) \psi_{1}(1)}{\lambda_{1}}+\frac{\psi_{2}(0) \psi_{2}(1)}{\lambda_{2}}+\ldots
$$

$$
\frac{1}{2 \pi} \sum_{\rho} \sum \frac{\left(r r^{\prime}\right)^{-\frac{1}{2}}\left(n+\frac{1}{2}\right) \mathrm{J}_{n+\frac{1}{2}}(\rho r) \mathrm{J}_{n+\frac{1}{3}}\left(\rho r^{\prime}\right) \mathrm{P}_{n}(\cos \gamma)}{\rho^{2} \int_{0}^{a} r J_{n+\frac{1}{2}}^{2}(\rho r) d r}
$$

Kneser* examines this case in detail, and verifies that the repeated series

$$
-\frac{1}{2 \pi \sqrt{r r^{\prime}}} \underset{n}{\Sigma}\left(n+\frac{1}{2}\right) \mathrm{P}_{n}(\cos \gamma) \sum_{\rho} \frac{J_{n+1}(\rho r)}{\rho^{2} \int_{0}^{\pi} r J_{n+k}^{2}(\rho r) d r}
$$

is equal to the known Green's Function.

\$13. Space bounded by the sphere $r=a$ and the cone $\theta=\theta_{0}$.

Here the normalised function

$$
\psi_{\rho m n}=\sqrt{\frac{a_{m}}{2 \pi r}} \frac{\mathrm{J}_{n+\frac{1}{2}}(\rho r) \mathrm{P}_{n}^{-m}(\mu)_{\sin ^{\cos } m \phi}^{\cos }}{\left[\int_{0}^{a} r \mathrm{~J}_{n+\xi}^{2}(\rho r) d r \int_{\mu_{0}}^{1}\left[\mathrm{P}_{n}^{-m}(\mu)\right]^{2} d \mu\right]^{\frac{1}{2}}} ;
$$

\footnotetext{
* Kneser, Integralgleichungen, \$38.
} 
$m$ being any positive integer, or zero :

$n$ any positive root of $\mathrm{P}_{n}^{-m}\left(\mu_{0}\right)=0$ :

$\rho$ any positive root of $\mathrm{J}_{n+1}(\rho a)=0$ :

$\lambda=\rho^{2}$, and $a_{0}=1, a_{m}=2$ for $m \geq 1$.

Thus the series corresponding to

is

$$
\frac{\psi_{1}(0) \psi_{1}(1)}{\lambda_{1}}+\frac{\psi_{2}(0) \psi_{2}(1)}{\lambda_{2}}+\ldots
$$

$$
\sum_{\rho m} \sum_{n} \frac{a_{n}}{2 \pi \sqrt{r r^{\prime}}} \frac{J_{n+\frac{1}{2}}(\rho r) J_{n+1}\left(\rho r^{\prime}\right)}{\rho^{2} \int_{0}^{a} r J_{n+1}^{2}(\rho r) d r} \frac{\mathbf{P}_{n}^{-m}(\mu) \mathbf{P}_{n}^{-m}\left(\mu^{\prime}\right)}{\int_{\mu_{0}}^{1}\left[\mathbf{P}_{n}^{-m}(\mu)\right]^{2} d \mu} \cos m\left(\phi-\phi^{\prime}\right) .
$$

If we sum first with regard to $\rho$, and use the results of $\S 6(I)$ and $\S 11(\mathrm{I})$, we obtain

$$
-\frac{1}{2 \pi \sqrt{r r^{\prime}}\left(1-\mu_{0}^{2}\right)} \sum_{m} a_{m} \cos m\left(\phi-\phi^{\prime}\right) \sum_{n}\left(\frac{r}{a}\right)^{n+\frac{1}{2}} \frac{\left(\frac{a}{r^{\prime}}\right)^{n+\frac{1}{3}}-\left(\frac{r^{\prime}}{a}\right)^{n+\frac{1}{2}}}{\frac{d}{d n} \mathrm{P}_{n}^{-m}\left(\mu_{0}\right) \frac{d}{d \mu_{0}} \mathrm{P}_{n}^{-m}\left(\mu_{0}\right)} \mathrm{P}_{n}^{-m}(\mu) \mathrm{P}_{n}^{-m}\left(\mu^{\prime}\right) .
$$

Collorary. Put $a=\infty$ in (18) and we find that in the cone $\theta=\theta_{0}$

$$
\mathrm{K}(0,1)=-\frac{1}{2 \pi \sqrt{r r^{\prime}}\left(1-\mu_{0}^{2}\right)} \sum_{m} a_{m} \cos m\left(\phi-\phi^{\prime}\right) \sum_{n}\left(\frac{r}{r^{\prime}}\right)^{n+\frac{1}{2}} \frac{\mathbf{P}_{n}^{-m}(\mu) \mathbf{P}_{n}^{-m}\left(\mu^{\prime}\right)}{\frac{d}{d n} \mathbf{P}_{n}^{-m}\left(\mu_{0}\right) \frac{d}{d \mu_{0}} \mathbf{P}_{n}^{-m}\left(\mu_{0}\right)} \text { for } r<r^{\prime}
$$

This agrees with Dougall, loc. cit. $\$ 29(2)$.

$\$ 14$. Space bounded by the sphere $r=a$, the cone $\theta=\theta_{0}$, and the planes $\phi=0, \phi=\alpha$.

$$
\text { Here } \psi_{\rho m n}=A_{\rho m n} \frac{J_{n+\frac{1}{2}}(\rho r)}{\sqrt{r}} P_{n}^{-\frac{m \pi}{a}}(\mu) \sin \frac{m \pi}{\alpha} \phi,
$$

where $m$ is any positive integer :

$n$ is any positive root of $P_{n}^{-\frac{m \pi}{a}}\left(\mu_{0}\right)=0$ :

$\rho$ is any positive root of $\mathrm{J}_{n+l}(\rho a)=0$ :

and $\lambda=\rho^{2}$.

" Cf. Macdonald, Demonstration of Green's Formula for Electric Density near the Vertex of a Right Cone, Trans. Camb. Phıl. Soc., Vol. XVIII., p. 293, 1900. The Green's Function for $\theta^{\prime}=0$ is found in that paper. 
We are thus led to the series

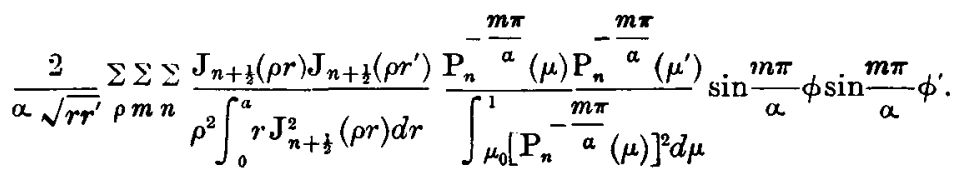

If we sum first with regard to $\rho$, using $\S 6$ (I.) and $\S 11$ (I.) we have

$$
\begin{array}{r}
-\frac{2}{\alpha \sqrt{r r^{\prime}}\left(1-\mu_{0}{ }^{2}\right)^{m}} \sum_{m} \sin \frac{m \pi}{\alpha} \phi \sin \frac{m \pi}{\alpha} \phi^{\prime} \sum_{n}\left(\frac{r}{a}\right)^{n+1}\left[\left(\frac{a}{r^{\prime}}\right)^{n+\frac{1}{2}}-\left(\frac{r^{\prime}}{a}\right)^{n+1}\right] \\
\frac{\mathrm{P}_{n}^{-m}(\mu) \mathrm{P}_{n}^{-m}\left(\mu^{\prime}\right)}{\frac{d}{d n} \mathrm{P}_{n}^{-m}\left(\mu_{0}\right) \frac{d}{d \mu_{0}} \mathrm{P}_{n}^{-m}\left(\mu_{0}\right)} \\
\text { for } 0<r<r^{\prime}<a . \quad(20)
\end{array}
$$

Corollary (i.) Put $a=\infty$ in (20) and we find for the space bounded by the cone $\theta=\theta_{0}$ and the two planes $\phi=0$ and $\phi=\alpha$,

$$
\mathrm{K}(0,1)=-\frac{2}{\alpha \sqrt{r r^{\prime}}\left(1-\mu_{0}^{2}\right) m} \sum_{m} \sin \frac{m \pi}{\alpha} \phi \sin \frac{m \pi}{\alpha} \phi^{\prime} \sum_{n}\left(\frac{r}{r^{\prime}}\right)^{n+\frac{1}{2}} \frac{\mathrm{P}_{n}^{-\frac{m \pi}{a}}(\mu) \mathrm{P}_{n}^{-\frac{m \pi}{a}}\left(\mu^{\prime}\right)}{\frac{d}{d n} \mathrm{P}_{n}^{-\frac{m \pi}{a}}\left(\mu_{0}\right) \frac{d}{d \mu_{0}} \mathrm{P}_{n}^{-\frac{m \pi}{a}}\left(\mu_{0}\right)}
$$

There is an obvious error in Dougall's result, loc. cit. $\$ 33(2)$, $\mathrm{S}_{n}^{m}(\beta)$ being omitted.

Corollary (ii.) Let $\theta_{0} \rightarrow \pi$ and we find from (21), with the aid of $\$ 11$ (II.), (IV.) for the space bounded by the planes $\phi=0, \phi=\alpha$,

$$
\mathrm{K}(0,1)=\frac{1}{\alpha \sqrt{r r^{\prime} m}} \sum_{\ln } \sin \frac{m \pi}{\alpha} \phi \sin \frac{m \pi}{\alpha} \phi^{\prime} \sum_{s} \frac{\Pi\left(s+2 \frac{m \pi}{\alpha}\right)}{\Pi(s)}\left(\frac{r}{r^{\prime}}\right)^{\frac{m \pi}{a}+s+\frac{1}{2}} \mathrm{P}_{\frac{m \pi}{a}+8}^{-\frac{m \pi}{a}}(\mu) \mathrm{P}_{\text {for } r<r^{\prime}, \quad(22}^{\frac{m \pi}{a}+s}\left(\mu^{\prime}\right)
$$

$s$ being any positive integer, including zero.

This agrees with Dougall, loc. cit. $\S 30$ (3).

\$14. Space bounded by two spheres $r=a, r=b$.

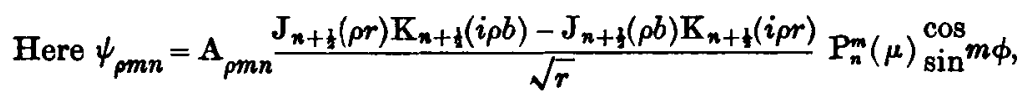


where $m$ is any positive integer, including zero :

$n$ is any positive integer :

$\rho$ is any positive root of $\mathrm{J}_{n+1}(\rho a) \mathrm{K}_{n+\frac{1}{3}}(i \rho b)$

and $\lambda=\rho^{2}$.

$$
-\mathbf{J}_{n+1}(\rho b) \mathbf{K}_{n+1}(i \rho a)=0 \text { : }
$$

We are thus led to the series

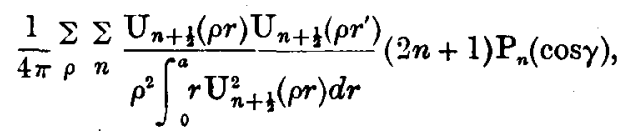

writing, as in $\S 6, \mathrm{U}_{n}(\rho x)$ for $\mathrm{J}_{n}(\rho x) \mathrm{K}_{n}(i \rho b)-\mathrm{J}_{n}(\rho b) \mathrm{K}_{n}(i \rho x)$.

If we sum first with regard to $\rho$, using $\S 6$ (II.) and put $r=a e^{\eta}, r^{\prime}=a e^{\eta^{\prime}}$, and $b=a e^{c}$, we obtain

$\frac{1}{2 \pi \sqrt{r r^{\prime}}} \Sigma \sinh \left(n+\frac{1}{2}\right) \eta \frac{\sinh \left(n+\frac{1}{2}\right)\left(c-\eta^{\prime}\right)}{\sinh \left(n+\frac{1}{2}\right) c} \mathbf{P}_{n}(\cos \gamma)$ for $\eta<\eta^{\prime}$.

This agrees with Dougall, loc. cit. $\S 28(1)$.

$\$ 15$. Space bounded by two spheres $r=a, r=b$, and the cone $\theta=\theta_{0}$.

Here $\psi_{\rho m n}=\mathbf{A}_{\rho m n} \frac{\mathrm{U}_{n+\frac{1}{3}(\rho r)}}{\sqrt{r}} \mathrm{P}_{n}^{-\frac{m \pi}{a}}(\mu)_{\sin }^{\cos } m \phi$,

where $m$ is any positive integer, including zero:

$n$ is any positive root of $\mathrm{P}_{n}{ }^{-\frac{m \pi}{a}}\left(\mu_{0}\right)=0$ :

$\rho$ is any positive root of $U_{n+\frac{1}{3}}(\rho a)=0$ :

and $\lambda=\rho^{2}$.

On normalising the function, and proceeding as in $\$ 14$, we obtain

$$
\begin{aligned}
-\frac{1}{\pi \sqrt{r r^{\prime}}} \Sigma a_{m} \cos m\left(\phi-\phi^{\prime}\right) \sum_{n} \frac{\sinh \left(n+\frac{1}{2}\right) \eta \sinh \left(n+\frac{1}{2}\right)\left(c-\eta^{\prime}\right)}{\sinh \left(n+\frac{1}{2}\right) c} \\
\frac{\mathrm{P}_{n}^{-\frac{m \pi}{a}}(\mu) \mathrm{P}_{n}^{-\frac{m \pi}{a}}\left(\mu^{\prime}\right)}{\left(1-\mu_{0}^{2}\right) \frac{d}{d n} \mathrm{P}_{n}^{-\frac{m \pi}{a}}\left(\mu_{0}\right) \frac{d}{d \mu_{0}} \mathrm{P}_{n}^{-\frac{m \pi}{a}}\left(\mu_{0}\right)},
\end{aligned}
$$

where $a_{0}=1, a_{m}=2$ for $m \geqq 1$ and $\eta<\eta^{\prime}$.

This is equivalent to Dougall's result, loc. cit. $\$ 31(2)$. 
§16. Space bounded by two spheres, $r=a, r=b$; tus axial planes $\phi=0, \phi=\alpha$; and the cone $\theta=\theta_{0}$.

Here we are led to the series

$$
\frac{2}{\alpha \sqrt{r r^{\prime}}} \sum_{\rho} \sum_{m} \sum_{n} \frac{\mathrm{U}_{n+\frac{1}{3}}(\rho r) \mathrm{U}_{n+\frac{1}{3}}\left(\rho r^{\prime}\right)}{\rho^{2} \int_{a}^{b} r \mathrm{U}_{n+1}^{2}(\rho r) d r} \frac{\mathrm{P}_{n}^{-\frac{m \pi}{a}}(\mu) \mathrm{P}_{n}^{-\frac{m \pi}{a}}\left(\mu^{\prime}\right)}{\int_{\mu_{0}}^{1}\left[\mathrm{P}_{n}^{-\frac{m \pi}{a}}(\mu)\right]^{2} d \mu} \sin \frac{m \pi}{\alpha} \phi \sin \frac{m \pi}{\alpha} \phi^{\prime} .
$$

Where $m$ is any positive integer ;

$$
\begin{array}{ll} 
& n \text { is any positive root of } \mathrm{P}_{n}^{-\frac{m \pi}{a}}\left(\mu_{0}\right)=0 \text { : } \\
\text { and } \quad & \rho \text { is any positive root of } \mathrm{U}_{n+\frac{1}{3}}(\rho a)=0: \\
\lambda=\rho^{2} .
\end{array}
$$

Proceeding as in $\$ 15$ we obtain, for $\eta<\eta^{\prime}$,

$$
\begin{aligned}
& -\frac{4}{\alpha \sqrt{r r^{\prime}}} \Sigma_{m} \sin \frac{m \pi}{\alpha} \phi \sin \frac{m \pi}{\alpha} \phi^{\prime} \sum_{n} \frac{\sinh \left(n+\frac{1}{2}\right) \eta \sinh \left(n+\frac{1}{2}\right)\left(c-\eta^{\prime}\right)}{\sinh \left(n+\frac{1}{2}\right) c} \\
& \frac{\mathrm{P}_{n}^{-\frac{m \pi}{a}}(\mu) \mathrm{P}_{n}^{-\frac{m \pi}{a}}\left(\mu^{\prime}\right)}{\left(1-\mu_{0}{ }^{2}\right) \frac{d}{d n} \mathrm{P}_{n}^{-\frac{m \pi}{a}}\left(\mu_{0}\right) \frac{d}{d \mu_{0}} \mathbf{P}_{n}^{-\frac{m \pi}{a}}\left(\mu_{0}\right)},
\end{aligned}
$$

Corollary. Let $\theta_{0} \rightarrow \pi$, and from $\$ 11$ (II.) and (IV.) we obtain for the space between two spheres and two axial planes

$$
\begin{aligned}
& K(0,1)=\frac{2}{\alpha \sqrt{r r^{\prime}}} \sum_{m} \sin \frac{m \pi}{\alpha} \phi \sin \frac{m \pi}{\alpha} \phi^{\prime} \sum_{s} \frac{\sinh \left(\frac{m \pi}{\alpha}+s+\frac{1}{2}\right) \eta \sinh \left(\frac{m \pi}{\alpha}+s+\frac{1}{2}\right)\left(c-\eta^{\prime}\right)}{\sinh \left(\frac{m \pi}{\alpha}+s+\frac{1}{2}\right) c} \\
& \frac{\Pi\left(2 \frac{m \pi}{\alpha}+s\right)}{\Pi(s)} \mathrm{P}_{\frac{m \pi}{a}+s}^{-\frac{m \pi}{a}}(\mu) \mathrm{P}_{\frac{m \pi}{a}+s}^{-\frac{m \pi}{a}}\left(\mu^{\prime}\right)
\end{aligned}
$$

$s$ being any positive integer, including zero, and $\eta<\eta^{\prime}$.

\$17. Space bounded by two spheres $r=a, r=b$ : two cones $\theta=\theta_{0}, \theta=\theta_{1}$ : and two planes $\phi=0, \phi=\alpha$. 
Here we are led to the series

$\frac{2}{\alpha \sqrt{r r^{\prime}}} \sum \sum_{\rho m} \sum_{n} \frac{U_{n+1}(\rho r) U_{n+1}\left(\rho r^{\prime}\right)}{\rho^{2} \int_{a}^{b} r U_{n+1}^{2}(\rho r) d r} \frac{\frac{m \pi}{S_{n}^{a}(\mu)} \frac{S_{n} \frac{m \pi}{a}}{\left(\mu^{\prime}\right)}}{\int_{\mu_{1}}^{\mu_{0}}\left[S_{n} \frac{m \pi}{a}(\mu)\right]^{2} d \mu} \sin \frac{m \pi}{\alpha} \phi \sin \frac{m \pi}{a} \phi^{\prime}$.

where

$m$ is any positive integer :

$n$ is any positive root of $P_{n}^{-\frac{m \pi}{a}}\left(\mu_{1}\right) P_{n}^{\frac{m \pi}{a}}\left(\mu_{0}\right)-P_{n}^{-\frac{m \pi}{a}}\left(\mu_{0}\right) P_{n}^{\frac{m \pi}{a}}\left(\mu_{1}\right)=0$, $\rho$ is any positive root of $U_{n+\frac{1}{2}}(\rho a)=0$ :

$\mathrm{S}_{n}^{m}(\mu)$ denotes $\mathrm{P}_{n}^{-\frac{m \pi}{a}}(\mu) \mathrm{P}_{n}^{\frac{m \pi}{a}}\left(\mu_{0}\right)-\mathrm{P}_{n}^{\frac{m \pi}{a}}(\mu) \mathrm{P}_{n}^{-\frac{m \pi}{a}}\left(\mu_{0}\right):$

and $\lambda=\rho^{2}$.

Using $\$ 6(\mathrm{II}$.$) and \$ 11(\mathrm{~V}$.$) , and summing first with regard$ to $\rho$, we obtain

$$
\begin{gathered}
\frac{4}{\left(1-\mu_{0}^{2}\right) a \sqrt{r r^{\prime}}} \sum \sin \frac{m \pi}{\alpha} \phi \sin \frac{m \pi}{\alpha} \phi^{\prime} \sum_{n} \frac{\sinh \left(n+\frac{1}{2}\right) \eta \sinh \left(n+\frac{1}{2}\right)\left(c-\eta^{\prime}\right)}{\sinh \left(n+\frac{1}{2}\right) c} \\
\frac{\mathrm{S}_{n}^{\frac{m \pi}{\alpha}}(\mu) \mathrm{S}_{n}{ }^{\frac{m \pi}{a}}\left(\mu^{\prime}\right)}{\left[\frac{d}{d n} \mathrm{~S}_{n} \frac{m \pi}{a}(\mu) \frac{d}{d \mu} \mathrm{S}_{n} \frac{m \pi}{a}(\mu)\right]_{\mu_{0}}^{\mu_{1}}} \text { for } \eta<\eta^{\prime} .
\end{gathered}
$$

Dougall, loc. cit. $\$ 35(2)$ gives a different result. 